\title{
Protective Effect of S-Allyl Cysteine Against Neonatal Asthmatic Rats
}

Dose-Response:

An International Journal

October-December 2020:1-10

(C) The Author(s) 2020

Article reuse guidelines:

sagepub.com/journals-permissions

DOI: $10.1177 / 1559325820982189$

journals.sagepub.com/home/dos

\section{(SSAGE}

\author{
Li Jiang' ${ }^{\oplus}$, Yuning $\mathrm{Li}^{\prime}$, Fang Wang', Xindao Zhang', and Ruiping Zhao'
}

\begin{abstract}
S-Allyl cysteine (SAC), an organic compound and a natural constituent of Allium sativum, commonly known as garlic have been consumed in routine foods are known to possess various biological activities. Nevertheless, scientific evidence on the protective effect of SAC against neonatal asthmatic rats is not available. Hence, the present study aimed at investigating the anti-asthmatic activity of SAC in neonatal asthmatic rats using Wistar rats. The study conducted in 4 groups consists of normal control rats, asthma-induced, asthma animals administered with SAC $(25 \mathrm{mg} / \mathrm{kg})$, and SAC control. At the end of the experimental period, inflammatory cells in bronchoalveolar lavage fluid (BALF), inflammatory markers, fibrinogen level, activated partial thromboplastin time, coagulation factor activity, and histopathology were elucidated. The current investigation exhibits that SAC significantly reduced the total leukocytes, with restored fibrinogen level, and activated partial thromboplastin time. In addition, the levels of inflammatory cytokines such as TNF- $\alpha$ (tumor necrosis factor- $\alpha$ ), IL-6 (Interleukin 6), and IL-I $\beta$ have also attenuated in SAC treated animals. Furthermore, the mRNA expression levels of COX2 (cyclooxygenase-2), MCP-I (monocyte chemoattractant protein-I), RANTES (regulated upon activation, normal T cell expressed and secreted), and eotaxin were reduced in SAC treated animals. Treatment of rats with SAC significantly reduced inflammation and eosinophil infiltration in the lungs. These results suggest that SAC exert protection in neonatal asthmatic rats suffering from acute or chronic inflammation by inducing anti-inflammatory and cell-protective responses.
\end{abstract}

\section{Keywords}

S-allyl cysteine, neonatal asthma, inflammation

\section{Introduction}

Asthma is an airway allergic disease caused by various factors in the environment like dust, smoke, and pollen from flowers, other activities of farming and urbanization, genetic factors, and hemostatic imbalance or hyperimmune activity due to infiltration of eosinophils. It is characterized by airway inflammation and structural remodeling. ${ }^{1-3}$ People affected by asthma are mostly the poor in developing countries and also children who have an underdeveloped immune system. Asthma is a disease that cannot be cured and have to be continuously monitored and needed continuous intake of medicines. ${ }^{4}$ The time, frequency, and season cannot be predicted, and one has to be prepared for it. The economically weak cannot afford to spend on medicines for long and cannot abstain from their regular daily commercial activity due to asthma-related factors.

Most of the factors that are known and tested for asthma can be grouped into any combination of these factors such as immunological, genetic and environmental conditions. The infiltration of the airway passage with inflammatory cells has been a significant contributor to inflammation in asthma.
Eosinophils- supported by IL-13, IL-4 activated CD4+ Th2 cells, contribute to the pathophysiology of asthma. Asthma-associated increased mucus secretion in the airway passage and accumulation of it cause wheeze, cough and decreased air intake due to bronchial congestion and discomfort or tightness of the chest. They are, at present, treated with corticosteroids and $\beta$-2-agonists in the form of inhalers to reduce the inflammation in the airway and bronchial constriction. ${ }^{5,6}$ These corticosteroids have been good at continuous usage, and the patients get the same symptoms after

\footnotetext{
' Department of Pediatrics, The First Hospital of Lanzhou University, Lanzhou, China

Received 21 September 2020; received revised 16 November 2020; accepted 26 November 2020

Corresponding Author:

Li Jiang, Department of Pediatrics, The First Hospital of Lanzhou University, Lanzhou 730000, China.

Email: lijianglijiang03@gmail.com
} 
Table I. Oligonucleotides Used in This Study.

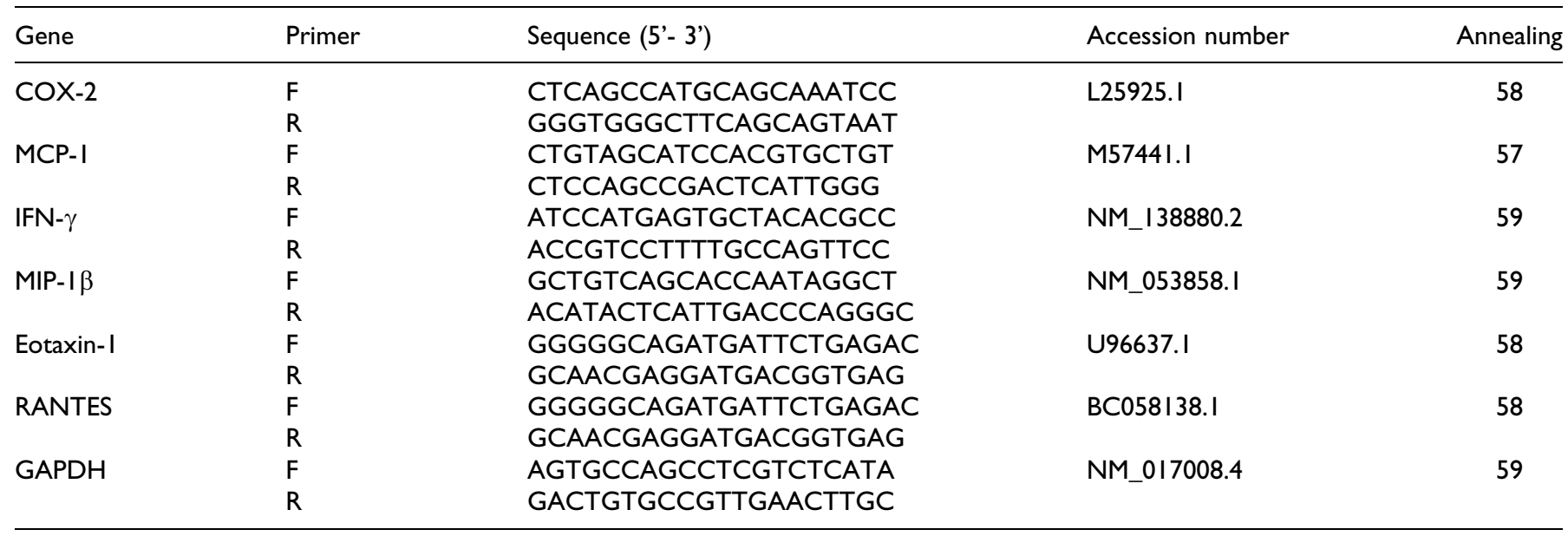

discontinuing the intake, and continuous usage would develop side effects. ${ }^{7-9}$ Hence, alternative molecules, preferable from natural sources, to control asthma without side effects and with long-lasting effects are needed.

Allium sativum, commonly known as garlic has been used in the food and the traditional medicines of Asia for centuries. It is rich in organosulfur compounds and has immunomodulatory properties, which can be used for health benefits besides its rich flavor and aroma for food preparation. Homoeopathic activities of garlic have been attributed to the aged garlic extracts, of which one of the organosulfur compound, S-allyl cysteine (SAC) has most of the medicinal value with antioxidant properties by scavenging the reactive oxygen species and reactive nitrogen species, ${ }^{10}$ increasing antioxidants ${ }^{11}$ and inhibiting prooxidant enzyme, cyclooxygenase. ${ }^{12}$ SAC has a half-life of more than $10 \mathrm{~h}^{13}$ after oral administration and gets easily absorbed in the gastrointestinal tract. ${ }^{14-16}$ Hence, the protective effect of SAC was known to be instant. In the current investigation, the importance of SAC in the context of alleviation of symptoms associated with asthma in neonatal rats was elucidated. The effect of SAC was illuminated from the data on decreasing the release of the inflammatory cytokines and inhibiting the airway inflammation and its development to lung fibrosis. We have used the ovalbumin (OVA)-induced asthmatic animal model to evaluate the use of SAC in the treatment of asthma.

\section{Materials and Methods}

\section{Experimental Groups and Induction Model}

Rat pups of Wistar strain, 12 days old were used in the present study. All animal research experiments were achieved as per the guidelines provided by the animal ethical committee, The First Hospital of Lanzhou University, Lanzhou, China. Prior permission was obtained from the Institutional Animal Care and Use Committee for the procedures implemented in the present study. The animals were housed in pathogen-free cages kept at the temperature of $20-25^{\circ} \mathrm{C}$ with $50-70 \%$ relative humidity $(\mathrm{RH})$. All rats were fed with clean tap water and commercial rat chow.

In the present study, rat pups were separated into 4 groups ( $\mathrm{n}=12$ in each group) as follows. The normal control group animals were administered with normal saline $(\mathrm{NaCl} 0.9 \%)$ for the sensitization, challenge, and drug-treated procedures instead of the respective treatments in other groups and considered as group 1. The group 2 animals were induced with asthma, for the induction of asthma in neonates, the previous publications were referred with slight modifications. ${ }^{17}$ Neonatal rats were sensitized to ovalbumin by inhalation of $0.75 \%$ (w/w) ovalbumin dissolved in saline using ultrasonic nebulization for 15 min daily for 45 days. While group 3 animals with asthma co-administered with S-allyl cysteine $(50 \mathrm{mg} / \mathrm{kg}$, oral administration), Group 4: S-allyl cysteine control, where the animals were administered with drug alone. At the end of the last ovalbumin challenge, the animals were killed by cervical decapitation, lung tissues were collected for the histological analysis, and blood samples were collected.

\section{Histological Analysis}

Lung tissues of control and experimental groups were isolated fixed in $10 \%$ formalin solution. The tissues were embedded in paraffin wax, and sections of $5 \mu \mathrm{m}$ thick slices were made using a microtome and stained with hematoxylin and eosin (H\&E).

\section{Assessment of Serum and Broncho-Alveolar Lavage Fluid (BALF)}

To determine the number of inflammatory cells in the BALF, the cells in the fluid were collected, stained with Wright's staining, and the differential cell count was estimated using a hemocytometer. On the other hand, the serum level of OVA-specific IgE and levels of nitric oxide (NO) was estimated using commercial ELISA kits as per the manufacturer's instructions (Wuhan Fine Biotech Co., Ltd., China). 


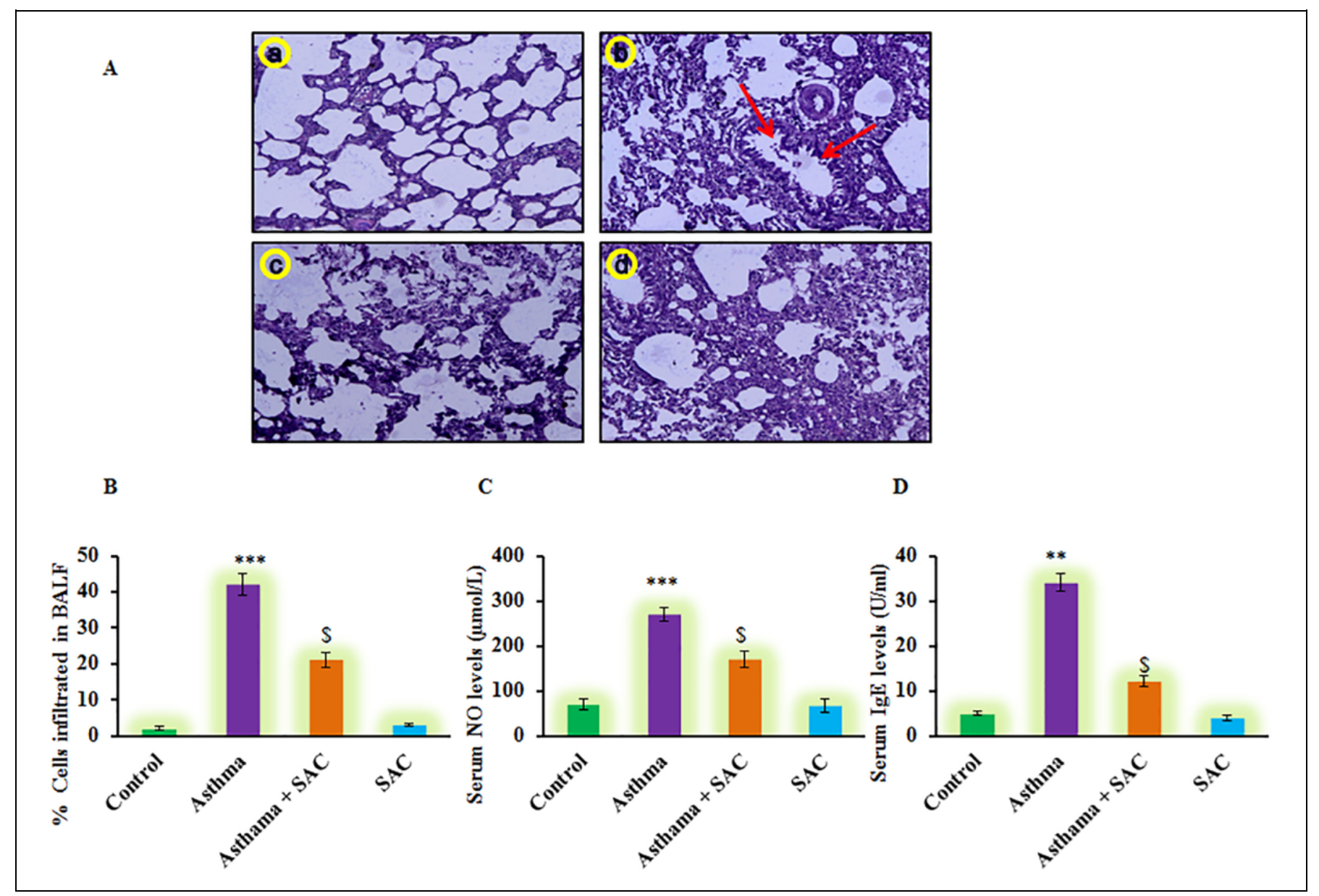

Figure I. (A) The lung tissue histology (a-d), (B-D) Represents the eosinophils infiltration in BALF; NO and IgE levels in serum of control and experimental group of rats. The experimental details were given in the methodology section. Values are expressed as mean \pm S.E $(n=8)$. Statistical significance expressed as ${ }^{* *} \mathrm{p}<0.0 \mathrm{I},{ }^{* * *} \mathrm{p}<0.00 \mathrm{I}$ compared to vehicle-treated controls, $\$ \mathrm{p}<0.05 \mathrm{SAC}$ compared to asthma rats; ns denotes non-significant.

\section{Determination of Blood Coagulation Time and Coagulation Factor Activity}

Fibrinogen level (FIB), prothrombin time (PT), thrombin time (TT), activated partial thromboplastin time (APTT), and the activity of coagulation factors were determined by standard methods.

\section{Estimation of Inflammatory Cytokines}

The assessment of pro-inflammatory and anti-inflammatory cytokines such as TNF- $\alpha$, IL-13, IL-17, IL-6, IL-10, and IL-1 $\beta$ in the serum samples and BALF samples was estimated using commercial ELISA kits as per the manufacturer's instruction. In addition, estimation of 8-isoprostane, prostaglandin E2, cysteinyl leukotrienes, and leukotriene B4 were also done using commercial kits (Wuhan Fine Biotech Co., Ltd., China).

\section{Reverse Transcription-PCR}

Total RNA was isolated from neonatal lung tissues using TRIzol reagent, and $20 \mu \mathrm{L}$ total RNA was converted into cDNA using a High-Capacity cDNA Reverse Transcription Kit. The real-time RT-PCR was done for specific genes using SYBR ${ }^{\circledR}$ Green PCR Kit, and the gene-specific primers used in the present study were listed in Table 1(Eurofins Genomics, Germany). The obtained $\mathrm{Ct}$ values were compared to determine the gene expressions by the comparative $\mathrm{Ct}$ method $(\Delta \Delta \mathrm{CT})$. The fold increase of the gene of interest was analyzed, keeping GAPDH as a control- house-keeping gene.

\section{Statistical Analysis}

Statistical significance was measured using Graph pad prism software. Differences between the groups with a p-value less than 0.05 were considered statistically significant.

\section{Results}

In the current investigation, the protective effect of SAC was evaluated in neonatal asthmatic rats. Figure 1 shows the histological analysis of experimental groups revealed that rats induced with asthma displayed the characteristic increase in smooth muscle mass, mucous gland hypertrophy, and vascular congestion 


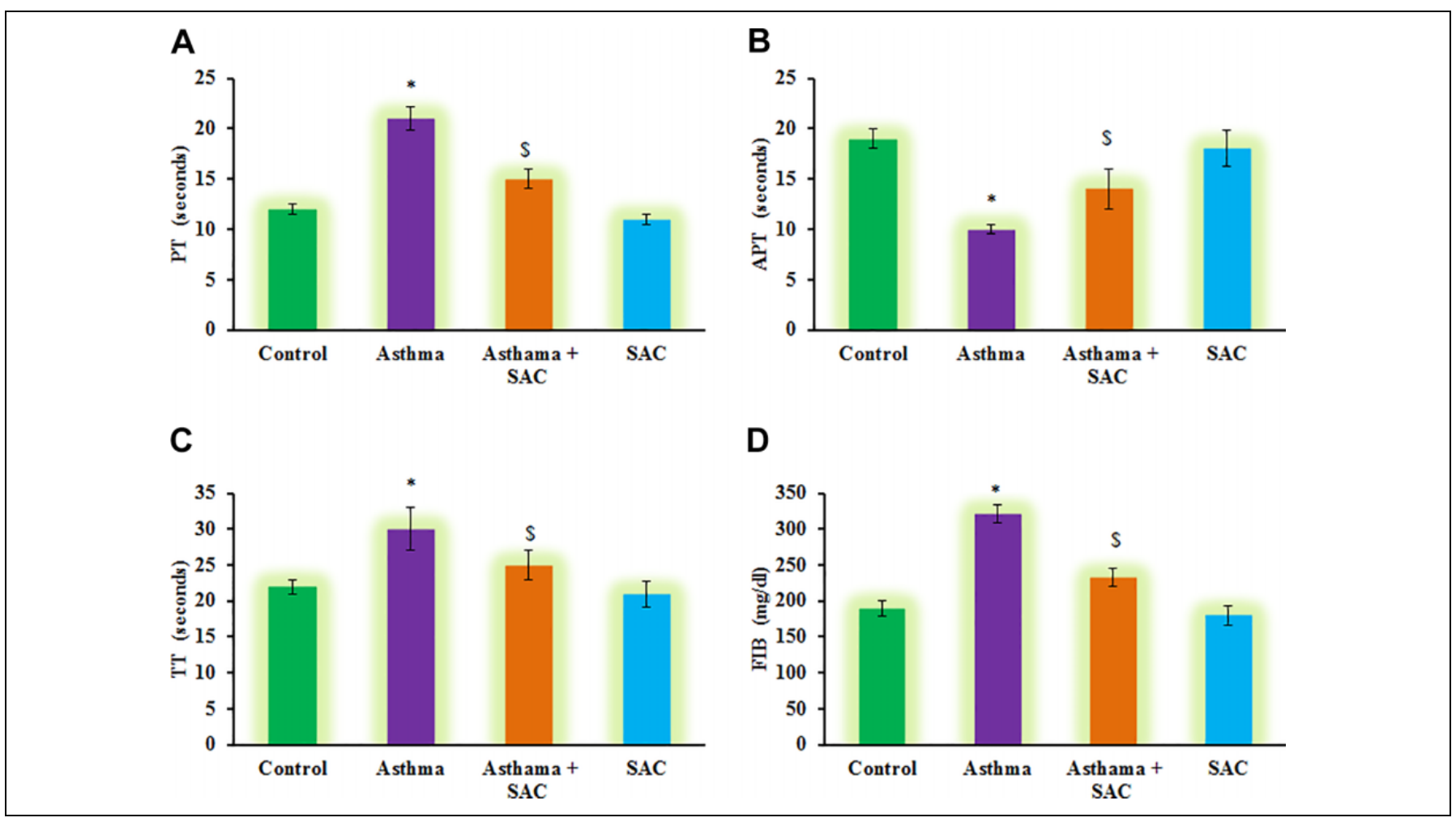

Figure 2. (A-D) represents the levels of renal PT, APT, TT, and FIB in the control and experimental group of rats. Values are expressed as mean \pm S.E $(n=8)$. Statistical significance expressed as $*_{p}<0.05$, compared to vehicle-treated controls, $\$ p<0.05$ SAC compared to asthma rats; ns denotes non-significant.

compared to control. While rats co-administered with SAC demonstrated a significant $(\mathrm{p}<0.01)$ reduction in hypertrophy development and smooth muscle mass (Figure 1A). Moreover, the leukocyte influx into BALF elicited a marked increase in the influx; the eosinophils constituted $<2.0 \%$ of total leukocytes in normal rats while the numbers were as high as $42 \%$ with higher levels of total IgE and NO levels compared to control rats. On the other hand, the SAC treatment considerably reduced the level of leukocyte infiltration and $\mathrm{NO}$ levels suggesting the protective role of SAC against asthma (Figure 1).

Furthermore, the estimation of PT, TT, APT, and FIB was carried out, and the results were presented in Figure 2. The results demonstrated that a significant reduction in the levels of PT, TT, APT, and FIB was found compared to control. However, these haematological parameters were found to be less affected in SAC treated rats compared to asthmatic animals (Figure 2). On the other hand, subsequent experiments to illuminate the cytokines levels were carried out, and the results are presented in Figure 3. In the current investigation, rats induced asthma demonstrated a significant increase in the levels of cytokines such as TNF- $\alpha(p<0.01)$, IL-13 ( $p<0.05)$, IL-17 $(\mathrm{p}<0.001)$, IL-6 $(\mathrm{p}<0.01)$, and IL-1 $\beta(\mathrm{p}<0.05)$ and decreased IL-10 ( $p<0.01)$ compared to control. While these inflammatory cytokines were diminished in SAC co-treatment, point out that $\mathrm{SAC}$ actively reduces the signaling of asthma progression is probably through abstaining the inflammatory molecules from getting into the signaling (Figure 3).
Also, to evaluate the condition of neonatal asthma, the markers of asthma induction such as 8-isoprostane, prostaglandin E2, cysteinyl leukotrienes, and leukotriene B4 were elucidated using ELISA method. From the above results on serum biochemical markers, the asthma onset was observed. Besides, the results demonstrated a significant increase $(\mathrm{p}<0.01)$ in the levels of 8-isoprostane, prostaglandin E2, cysteinyl leukotrienes, and leukotriene B4 levels in the asthma group compared to control. On the other hand, rats had SAC exposure, elicited the decreased levels of these indicator molecules shows SAC exerts protection (Figure 4).

To corroborate the role of SAC on the inflection of inflammatory signals, the mRNA levels of cytokines were elucidated about the control gene, and the results were presented in Figure 5 . The results demonstrated that a profound increase $(\mathrm{p}<0.01)$ in the mRNA transcript expression of $C O X-2$ (3.2-fold), $M C P-1$ (2.2-fold), IFN- $\gamma$ (4-fold), MIP-1 $\beta$ (3.3-fold), Eotaxin-1 (2.6-fold), RANTES (2.8-fold) levels in asthma-induced rats compared to control. However, the increased levels of these chemokines were found reduced in SAC co-treatment indicate the promising role of SAC in exerting protection against neonatal asthma (Figure 5).

\section{Discussion}

The chronic inflammatory disease, asthma is characterized by increased airway hyperresponsiveness to irritants such as smoke, dust, or other allergens causing wheezing, discomfort 


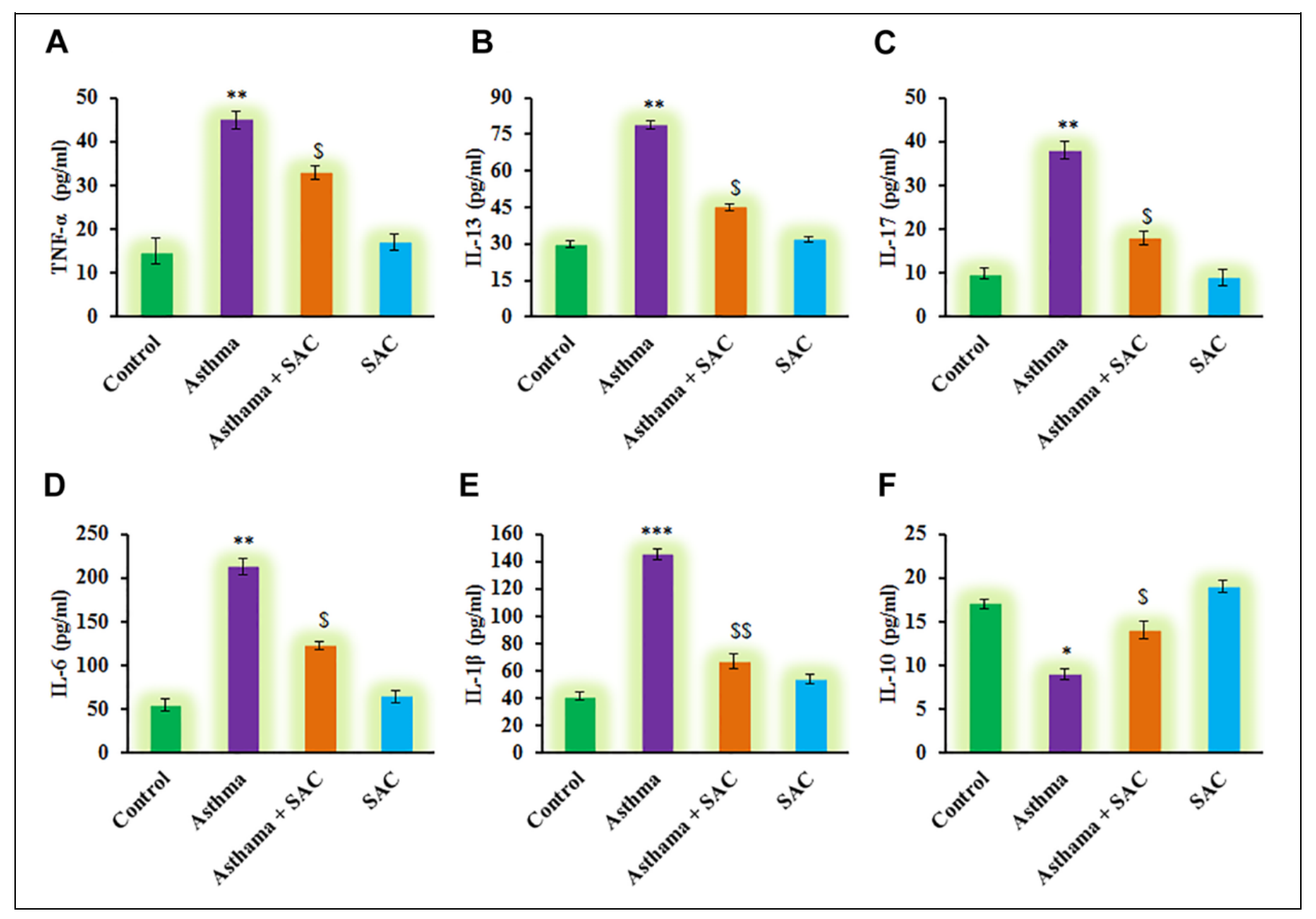

Figure 3. (A-F) represents cytokine expression analysis of TNF- $\alpha$, IL-I3, IL-I7, IL-6, IL-I $\beta$ and IL- I0 in the control and experimental group of rats. The experimental details were given in the methodology section. Values are expressed as mean \pm S.E $(n=8)$. Statistical significance expressed as $*_{p}<0.05$, $*_{p}^{*}<0.01$, $*^{* *} p<0.001$ compared to vehicle-treated controls, $\$ p<0.05, \$ \$ p<0.01$, SAC compared to asthma rats; ns denotes non-significant.

in the chest due to difficulty in breathing and mucus accumulation in the airway. ${ }^{18}$ The pathophysiology behind these symptoms is due to increased infiltration of mast cells, and eosinophils which cause inflammation in the airway due to activation of these immune cells by allergens. The activated immune cells thus cause an increase in mucus secretion, contributing to these symptoms. In our immuno-histological results, we have shown the severity of the onset of asthma in the OVA-induced asthmatic group, and such infiltration of inflammatory cells is absent, or the effects were less in the SAC-co-administered group. The impact of such a decrease in the infiltration was seen as decreased mucus secretion, ${ }^{19,20}$ and the symptoms associated in the aftermath of mucus secretion were also less. SAC relives the alveoli of the animals affected by asthma by decreasing the infiltration of eosinophils and monocytes into them and decreasing the number of goblet cells in the airway that is responsible for mucus secretion.

Infiltration of eosinophils, lymphocytes, and mast cells into the airways has been the hallmark of asthma. In order to find the extent of onset of inflammation in the OVA-induced asthmatic rats, we have tested the BALF for the eosinophils and mast cells concerning the disease severity and subsequent airway inflammation and mucus secretion due to hyperresponsiveness. ${ }^{21}$ These numbers were high in the OVA-induced asthmatic rats and got reduced with the treatment of SAC in the other group. Also, histological analysis of the pulmonary region indicates that SAC could inhibit the eosinophil numbers in the airway and hence the inflammation. Such results would improve the reliability of SAC in its use against asthma and airway inflammation.

Asthma has been well associated with the hemostatic imbalance of Th1/Th2, and TNF- $\alpha$ expression is observed. ${ }^{22}$ Mice studies have indicated that anti-TNF- $\alpha$ antibodies have reduced the intensity of antigen-induced airway hyperresponsiveness and inflammation due to eosinophilic infiltration of the airway. ${ }^{23,24} \mathrm{TNF}-\alpha$ produced by eosinophils and T-cells effectively damages the airway by activating the expression of adhesion molecules for the sustained presence of eosinophils in the airways. ${ }^{25} \mathrm{TNF}$-alpha also increases the goblet cell metaplasia to increase the excretion of mucus in the lungs. ${ }^{26}$ These 


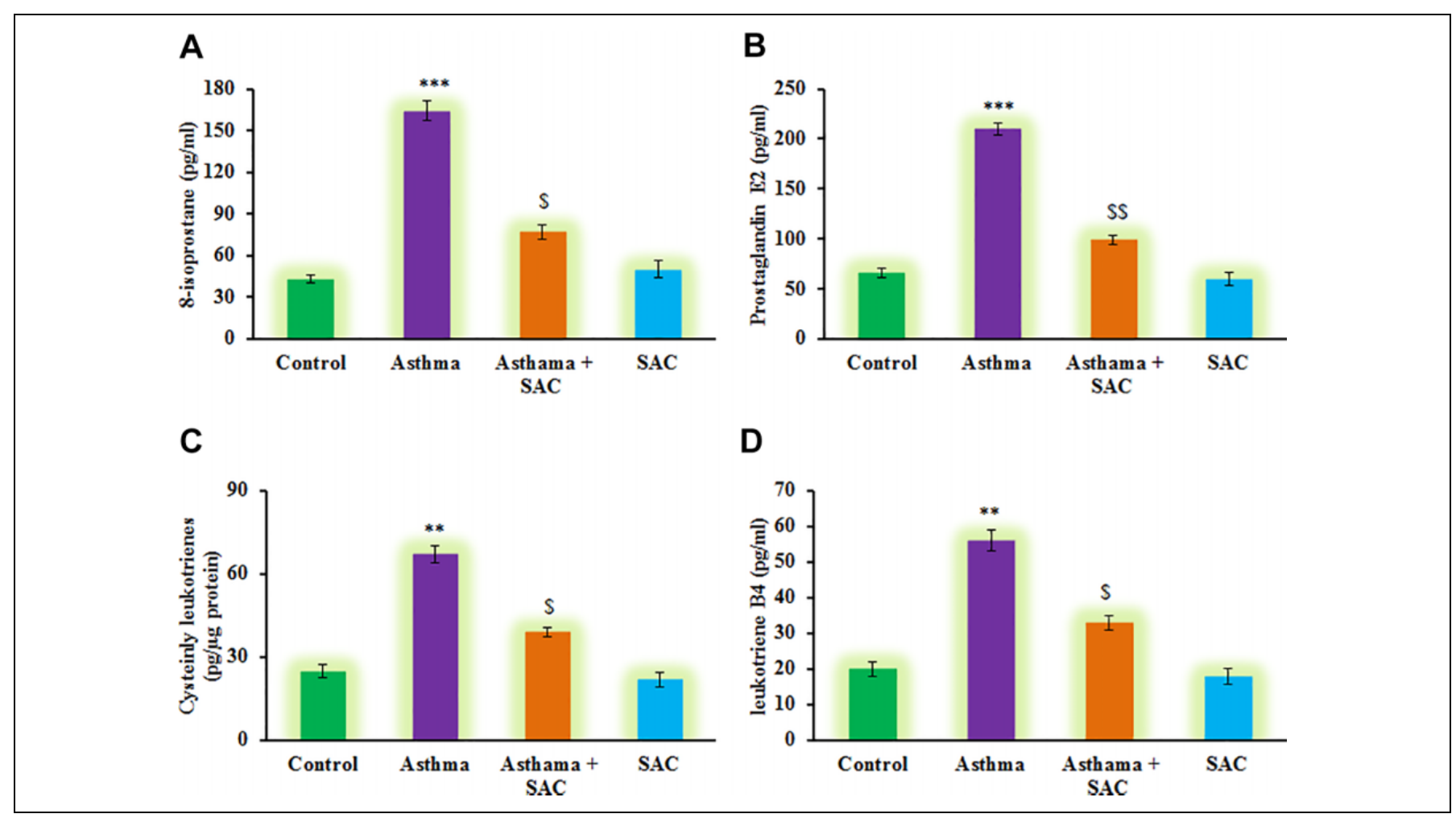

Figure 4. (A-D) represents the analysis of inflammatory molecules such as 8-isoprostane, prostaglandin E2, cysteinyl leukotrienes and leukotriene B4 in the control and experimental group of rats. The experimental details were given in the methodology section. Values are expressed as mean \pm S.E $(n=8)$. Statistical significance expressed as $* * p<0.01$, ***p $<0.001$ compared to vehicle-treated controls, $\$ p<0.05$, $\$ \$ p<0.0$ I SAC compared to asthma rats; ns denotes non-significant.

effects observed in our asthmatic rats were reduced when SAC was co-administered in the OVA-induced asthmatic rats. Infiltration of inflammatory cells would generate $\mathrm{ROS},{ }^{27}$ and increased eosinophils have produced superoxide anions to react with nitric oxide ${ }^{28}$ and cause oxidative stress in the affected animals. Hence the NO levels were higher in the asthmatic animals and got reduced in their levels in SAC-co-administered animals. A typical Th2-type immune response was observed in asthmatic animals, which is the increased expression of $\operatorname{IgE}$ against OVA. ${ }^{29}$

In the development and spreading of allergic inflammation, the hemostatic imbalance is important and can be detected from fibrinogen level (FIB), prothrombin time (PT), thrombin time (TT), activated partial thromboplastin time (APTT). The OVA-induced asthma group has significantly higher FIB and $\mathrm{TT}$, and the trends were reversed in the SAC-co-administered rats. Thus the hemostatic imbalance in the OVA-induced rats was reversed in the treated group. Also, the coagulation factors activity was tested in all the groups indicating the asthmatic group had higher FXII activity and decreased in the activity of FII and FV factors. Fibrinogen, generated through airway inflammation, would impair the hemostatic imbalance, and such observations were not present in the SAC-group.

We observed an increase in the expression of IL-13 in the BALF, peripheral blood, mast cells in the OVA-induced asthmatic animals, and is responsible for the inflammation in the airway, remodeling, and acute hyperresponsiveness. ${ }^{30,31}$ IL-13 plays a major role in the pathogenesis of acute hyper-responsiveness ${ }^{32,33}$ by increasing the mucous secretion through goblet cell metaplasia. ${ }^{34}$ Such observa6tion has well correlated with the number of eosinophils observed in the asthmatic animals, without treatment, that is having airway inflammation ${ }^{35}$ as overexpression of IL-13 is very important for the survival of eosinophils, their activation ${ }^{34}$ and chemotaxis the eosinophils to the site of inflamed or damaged airway tissues. ${ }^{36}$ Our OVA-induced animal models showed the eosinophil infiltration, airway inflammation, mucus secretion, and hyperresponsiveness, which were not observed with the SAC-co-administered OVA models. Hence, IL-13 is essential in playing a major role in the pathogenesis of allergen-induced asthma, and SAC is inhibiting primarily the airway inflammation, which is a probable small molecule candidate to be used similar to the inhibition of eosinophil inflammation as observed here. ${ }^{30}$

IL-17 is significantly higher in the airway of asthmatic animals, which is similar to the mice experimental results of asthma, which were induced with OVA. ${ }^{37}$ It is present at a level that is significant when compared to the normal animals in our experimental animal groups and is characteristic of the onset of severe asthma. ${ }^{38,39}$ An increase in the airway inflammation of OVA-induced rats has been partially attributed to IL-17 since the implication of overexpression of IL-13 in this model has a deep impact on the airway inflammation and hold 


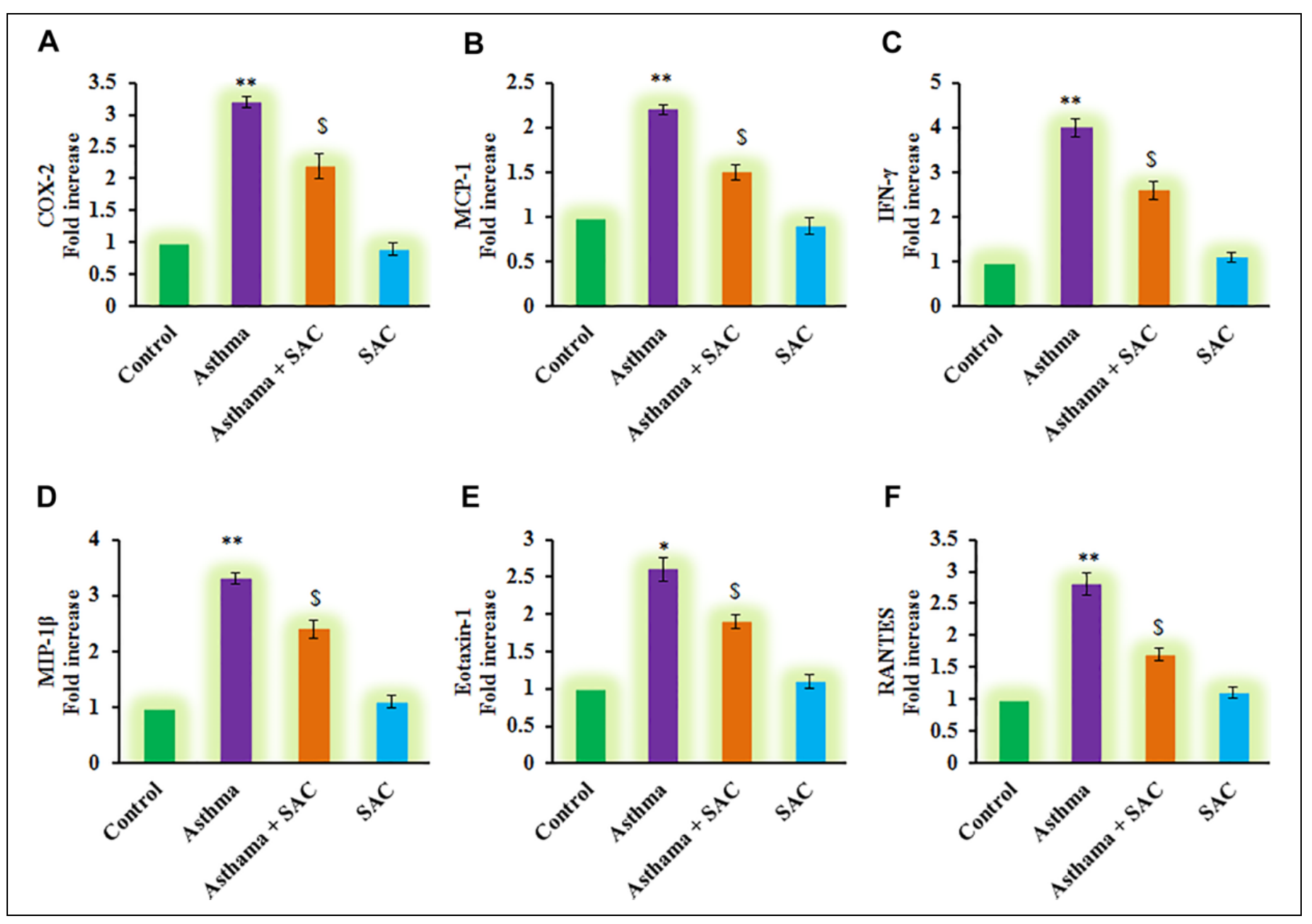

Figure 5. (A-F) represents qRT-PCR mRNA expression analysis of COX-2, MCP-I, IFN- $\gamma$, MIP-I $\beta$, Eotaxin- I, RANTES in control and experimental group of cells. The qRT-PCR experimental details were given in the methodology section. Values are expressed as mean \pm S.E $(n=8)$. Statistical significance expressed as ${ }^{*} p<0.05$, ${ }^{*} \mathrm{p}<0.01$ compared to vehicle-treated controls, $\$ \mathrm{p}<0.05$ SAC compared to asthma rats; ns denotes nonsignificant.

prime responsibility in the airway inflammation of lung in asthma-induced rats as the route of OVA was induced through inhalation and not through the skin. This is in-line with the skin-induced OVA induction observed in research done here, ${ }^{37}$ where the infiltration of IL-17 secreting cells would occur in the lung only in the absence of expression of IL-13.

With the administration of allergens, the airway epithelium is infected and has undergone inflammation, ${ }^{40,41}$ and elevated expression of IL-6 in the lungs is observed. ${ }^{42}$ IL-6 is known to be a pro-inflammatory mediator and is involved in the synthesis of prostaglandin E2 (PGE2) and promotes the infiltration of eosinophils in the airway. The expression of $C O X-2$ high in asthmatic animals indicates that the inflammatory cytokine, IL-6 would mediate the immune cells to generate PGE $2 .{ }^{43}$ The levels of IL-6 are increased in our asthmatic groups of animals, and the animals are known to be clinically asthmatic. ${ }^{44}$ IL-6 also promotes IL-13 production ${ }^{42}$ in asthmatic animals, and such synergistic action has been observed in human patients who are allergic. The co-administration of SAC has decreased the IL-6 levels and also IL-13 indicating that such effects are not due to complete blockage of IL-6 R but by increasing the expression of Treg cells and reducing expression of CD4 T effector cells. ${ }^{45,46}$

The simultaneous increase in the expression of IL-1beta indicates that IL-1beta, along with IL-6, would aid in the differentiation of Th17 cells ${ }^{47}$ that contribute to the airway inflammation in asthmatic allergies. ${ }^{48,49}$ Such events did not occur in our SAC-coadministered rats, where the airway inflammation was nullified. Lung epithelial cells could not produce IL-6 due to decreased IL-17 in our SAC-group as in, and hence no positive feedback mechanism ${ }^{44}$ occurred to raise IL- 6 and hence the sequence of inflammatory events triggered by IL-13, goblet cell activation, mucus production, hyperresponsiveness, and airway inflammation. Treatment of SAC has increased the anti-inflammatory molecule IL-10 in effectively controlling the inflammation.

The consequence of inflammation in the airway would be the oxidant damage with the assistance of antioxidants and reactive oxygen species (ROS) having primary role thereafter in sustaining the inflammation ${ }^{50}$ to cause tissue damage. ${ }^{51}$ 
Accumulation of ROS would lead to peroxidation of arachidonic acid to produce many isomers of isoprostanes, of which, 8 -isoprostane is involved in the constriction of smooth muscles and airway obstruction ${ }^{52}$ that cause discomfort in breathing during asthma. Leukotriene members such as cysteinyl leukotrienes, leukotriene B4, also derived from arachidonic acid by another pathway. ${ }^{53}$ In eosinophils, act as potent broncho constrictors and causing airway smooth muscle constriction and increased mucous secretion. The increase in such lipid peroxidation products has been observed in naïve children ${ }^{54}$ alike in our neonatal rats induced with OVA rather than in other subjects. The reduction in the levels of 8-isoprostanes in our SAC-co-administered animal model indicates that SAC would inhibit the inflammation, and the effect is long-lasting, unlike conventional corticosteroid therapy where residual inflammation $^{55}$ in asthmatic individuals resides.

To understand the cellular events and alterations occurring at the molecular level, due to IL-13, in the lungs, we have elucidated the levels of chemokines that are stimulated by IL-13. ${ }^{56}$ In that order, we have checked the levels of $M C P-1$, $M I P-1 \beta, R A N T E S$, and eotaxin in the lungs. IL-13 plays as an effector in the Th2 inflammation ${ }^{57}$ and regulates the inflammation into the remodeling of the lung tissues ${ }^{58,59}$ and thus contributing to hyper-responsiveness. ${ }^{60-62}$ The coordinated response of the various chemokines such as eotaxin, RANTES, $M I P 1-\beta$, and $M C P-1$ results in the Th2 inflammatory response in the affected lungs which would eventually increase trafficking of eosinophils from the blood circulation into the airway, ${ }^{63}$ upregulating the adhesion molecules, to attach them to the airway epithelial cells. We did not necessarily discuss the molecular mechanism into the activation of Th2 immune response in asthma, but we speculate that the expression of chemokines with IL-13 activation would be to enhance the pre-effector events of sensitization, Th2 cell generation, and Th2 cytokine elaboration in the airway inflammation. ${ }^{56}$

\section{Conclusion}

In summary, the present study demonstrates that SAC is a potent drug candidate to inhibit the airway inflammation by decreasing the expression of various inflammatory cytokines, in cases where even corticosteroids have failed to give an improvement in the treatment. It is implicated in the pathogenesis of airway inflammation in OVA-induced asthma, and interleukins play as a stimulator of various cytokines and chemokines to attract the eosinophils and neutrophils by chemotaxis into the airway to cause a respiratory burst and thereby increasing the inflammation and tissue injury which can be attenuated using SAC. However, the study has a shortcoming in that we have failed to address the molecular pathway that SAC undertakes in inhibiting the airway inflammation which future research would address.

\section{Declaration of Conflicting Interests}

The author(s) declared no potential conflicts of interest with respect to the research, authorship, and/or publication of this article.

\section{Funding}

The author(s) disclosed receipt of the following financial support for the research, authorship, and/or publication of this article: This study was supported by the Natural Science Foundation of Beijing, China (Grant Number: 18JR3RA357).

\section{ORCID iD}

Li Jiang (D) https://orcid.org/0000-0001-9866-3950

\section{References}

1. Wills-Karp M. Immunologic basis of antigen-induced airway hyperresponsiveness. Annu Rev Immunol. 1999;17:255-281.

2. Elias JA, Lee CG, Zheng T, Ma B, Homer RJ, Zhu Z. New insights into the pathogenesis of asthma. J Clin Invest. 2003; 111(3):291-297.

3. Regal JF. Immunologic effector mechanisms in animal models of occupational asthma. J Immunotoxicol. 2004;1(1):25-37.

4. Calabria CW, Hauswirth DW, Rank M, Sher L. American academy of asthma, allergy \& immunology membership experience with venom immunotherapy in chronic medical conditions and pregnancy, and in young children. Allergy Asthma Proc. 2017;38(2):121-129.

5. Sheth A, Reddymasu S, Jackson R: Worsening of asthma with systemic corticosteroids. A case report and review of literature. $J$ Gen Intern Med. 2006;21(2):C11-13.

6. Walsh GM. An update on emerging drugs for asthma. Expert Opin Emerg Drugs. 2012;17(1):37-42.

7. Guilbert TW, Morgan WJ, Zeiger RS, et al. Long-term inhaled corticosteroids in preschool children at high risk for asthma. N Engl J Med. 2006;354(19):1985-1997.

8. Wenzel SE, Covar R. Update in asthma 2005. Am J Respir Crit Care Med. 2006;173(7):698-706.

9. Aun MV, Ribeiro MR, Costa Garcia CL, Agondi RC, Kalil J, Giavina-Bianchi P. Esophageal candidiasis - an adverse effect of inhaled corticosteroids therapy. J Asthma. 2009;46(4): 399-401.

10. Koch HP. The long path toward odorless garlic. Pharm Unserer Zeit. 1996 25(4):186-191.

11. Block E. The chemistry of garlic and onions. Sci Am. 1985; 252(3):114-119.

12. Sakamoto K, Lawson LD, Milner JA. Allyl sulfides from garlic suppress the in vitro proliferation of human A549 lung tumor cells. Nutr Cancer. 1997;29(2):152-156.

13. Kodera Y, Suzuki A, Imada O, et al. Physical, chemical, and biological properties of S-allyl cysteine, an amino acid derived from garlic. J Agric Food Chem. 2002;50(3):622-632.

14. Nagae S, Ushijima M, Hatono S, et al. Pharmacokinetics of the garlic compound S-allylcysteine. Planta Med. 1994;60(3): 214-217.

15. Liu Z, Li M, Chen K, et al. S-allyl cysteine induces cell cycle arrest and apoptosis in androgen-independent human prostate cancer cells. Mol Med Rep. 2012;5(2):439-443.

16. Syu JN, Yang MD, Tsai SY, et al. S-allylcysteine improves blood flow recovery and prevents ischemic injury by augmenting neovasculogenesis. Cell Transplant. 2017;26(10):1636-1647. 
17. Liu S, Shudou M, Maeyama K. Early activation of mucosal mast cells during the primary immune response in a rodent model of neonatal asthma. Immunol Cell Biol. 2011;89(2):239-245.

18. Lee MY, Seo CS, Ha H, et al. Protective effects of Ulmus davidiana var. japonica against OVA-induced murine asthma model via upregulation of heme oxygenase-1. J Ethnopharmacol. 2010; 130(1):61-69.

19. Bochner BS, Busse WW. Advances in mechanisms of allergy. J Allergy Clin Immunol. 2004;113(5):868-875.

20. Bochner BS, Busse WW: Allergy and asthma. J Allergy Clin Immunol. 2005;115(5):953-959.

21. Wu S, Yang R, Wang G. Anti-asthmatic effect of pitavastatin through aerosol inhalation is associated with CD4+ CD25+ Foxp3+ T cells in an asthma mouse model. Sci Rep. 2017;7(1): 6084.

22. Nam HS, Lee SY, Kim SJ, et al. The soluble tumor necrosis factor-alpha receptor suppresses airway inflammation in a murine model of acute asthma. Yonsei Med J. 2009;50(4):569-575.

23. Rudmann DG, Moore MW, Tepper JS, et al. Modulation of allergic inflammation in mice deficient in TNF receptors. $\mathrm{Am} \mathrm{J}$ Physiol Lung Cell Mol Physiol. 2000;279(6):L1047-1057.

24. Zuany-Amorim C, Manlius C, Dalum I, et al. Induction of TNFalpha autoantibody production by AutoVac TNF106: a novel therapeutic approach for the treatment of allergic diseases. Int Arch Allergy Immunol. 2004;133(2):154-163.

25. Gaga M, Lambrou P, Papageorgiou N, et al. Eosinophils are a feature of upper and lower airway pathology in non-atopic asthma, irrespective of the presence of rhinitis. Clin Exp Allergy. 2000;30(5):663-669.

26. Thomas PS. Tumour necrosis factor-alpha: the role of this multifunctional cytokine in asthma. Immunol Cell Biol. 2001;79(2): 132-140.

27. Sahiner UM, Birben E, Erzurum S, et al. Oxidative stress in asthma. World Allergy Org. 2011;J 4(10):151-158.

28. Barnes PJ, Kharitonov SA. Exhaled nitric oxide: a new lung function test. Thorax. 1996;51(3):233-237.

29. Ano S, Morishima Y, Ishii Y, et al. Transcription factors GATA-3 and RORgammat are important for determining the phenotype of allergic airway inflammation in a murine model of asthma. J Immunol. 2013;190(3):1056-1065.

30. Wills-Karp M, Luyimbazi J, Xu X, et al. Interleukin-13: central mediator of allergic asthma. Science. 1998;282(5397):2258-2261.

31. Zhu Z., Homer RJ, Wang Z, et al. Pulmonary expression of interleukin-13 causes inflammation, mucus hypersecretion, subepithelial fibrosis, physiologic abnormalities, and eotaxin production. J Clin Invest. 1999;103(6):779-788.

32. Brightling CE, Symon FA, Holgate ST, et al. Interleukin-4 and -13 expression is co-localized to mast cells within the airway smooth muscle in asthma. Clin Exp Allergy. 2003;33(12): 1711-1716.

33. Berry MA, Parker D, Neale N, et al. Sputum and bronchial submucosal IL-13 expression in asthma and eosinophilic bronchitis. J Allergy Clin Immunol. 2004;114(5):1106-1109.

34. Doran E, Cai F, Holweg CTJ, et al. Interleukin-13 in asthma and other eosinophilic disorders. Front Med. 2017;4:139.
35. Truyen E, Coteur L, Dilissen E, et al. Evaluation of airway inflammation by quantitative Th1/Th2 cytokine mRNA measurement in sputum of asthma patients. Thorax. 2006;61(3):202-208.

36. Wynn TA. IL-13 effector functions. Annu Rev Immunol. 2003;21: 425-456.

37. He R, Kim HY, Yoon J, et al. Exaggerated IL-17 response to epicutaneous sensitization mediates airway inflammation in the absence of IL-4 and IL-13. J Allergy Clin Immunol. 2009;124(4): 761-770. e761.

38. Wong CK, Ho CY, Ko FW, et al. Proinflammatory cytokines (IL-17, IL-6, IL-18 and IL-12) and Th cytokines (IFN-gamma, IL-4, IL-10 and IL-13) in patients with allergic asthma. Clin Exp Immunol. 2001;125(2):177-183.

39. Bullens DM, Truyen E, Coteur L, et al. IL-17 mRNA in sputum of asthmatic patients: linking $\mathrm{T}$ cell-driven inflammation and granulocytic influx? Respir Res. 2006;7:135.

40. Allard JB, Poynter ME., Marr KA, et al. Aspergillus fumigatus generates an enhanced Th2-biased immune response in mice with defective cystic fibrosis transmembrane conductance regulator. J Immunol. 2006;177(8):5186-5194.

41. Allard JB, Rinaldi L, Wargo MJ, et al. Th2 allergic immune response to inhaled fungal antigens is modulated by TLR-4independent bacterial products. Eur J Immunol. 2009;39(3): 776-788.

42. Neveu WA, Allard JB, Dienz O, et al. IL-6 is required for airway mucus production induced by inhaled fungal allergens. J Immunol. 2009;183(3):1732-1738.

43. Sousa A, Pfister R, Christie PE, et al. Enhanced expression of cyclo-oxygenase isoenzyme $2(\mathrm{COX}-2)$ in asthmatic airways and its cellular distribution in aspirin-sensitive asthma. Thorax. 1997; 52:940-945.

44. Rincon M, Irvin CG. Role of IL-6 in asthma and other inflammatory pulmonary diseases. Int J Biol Sci. 2012;8(9):1281-1290.

45. Doganci A, Eigenbrod T, Krug N, et al. The IL-6 R alpha chain controls lung $\mathrm{CD} 4+\mathrm{CD} 25+$ Treg development and function during allergic airway inflammation in vivo. J Clin Invest. 2005; 115(2):313-325.

46. Finotto S, Eigenbrod T, Karwot R, et al. Local blockade of IL-6 R signaling induces lung $\mathrm{CD} 4+\mathrm{T}$ cell apoptosis in a murine model of asthma via regulatory T cells. Int Immunol. 2007;19(6): 685-693.

47. Ghoreschi K, Laurence A, Yang XP, et al. Generation of pathogenic $\mathrm{T}(\mathrm{H}) 17$ cells in the absence of TGF-beta signalling. Nature. 2010;467(7318):967-971.

48. Nakae S, Nambu A, Sudo K, et al. Suppression of immune induction of collagen-induced arthritis in IL-17-deficient mice. J Immunol. 2003;171(11):6173-6177.

49. Schnyder-Candrian S, Togbe D, Couillin I, et al. Interleukin-17 is a negative regulator of established allergic asthma. J Exp Med. 2006;203(12):2715-2725.

50. Wood LG, Gibson PG, Garg ML. Biomarkers of lipid peroxidation, airway inflammation and asthma. Eur Respir. 2003;J 21(1): 177-186.

51. Doelman CJ, Bast A. Oxygen radicals in lung pathology. Free Radic Biol Med. 1990;9(5):381-400. 
52. Okazawa A, Kawikova I, Cui ZH, et al. 8-Epi-PGF2alpha induces airflow obstruction and airway plasma exudation in vivo. $\mathrm{Am} \mathrm{J}$ Respir Crit Care Med. 1997;155(2):436-441.

53. Bisgaard H: Leukotriene modifiers in pediatric asthma management. Pediatrics. 2001;107(2):381-390.

54. Zanconato S, Carraro S, Corradi M, et al. Leukotrienes and 8-isoprostane in exhaled breath condensate of children with stable and unstable asthma. J Allergy Clin Immunol. 2004;113(2):257-263.

55. Kharitonov SA, Donnelly LE, Montuschi P, et al. Dose-dependent onset and cessation of action of inhaled budesonide on exhaled nitric oxide and symptoms in mild asthma. Thorax. 2002;57(10):889-896.

56. Zhu Z, Ma B, Zheng T, et al. IL-13-induced chemokine responses in the lung: role of CCR2 in the pathogenesis of IL-13-induced inflammation and remodeling. J Immunol. 2002;168(6): 2953-2962.

57. Ma B, Zhu Z, Homer RJ, et al. The C10/CCL6 chemokine and CCR1 play critical roles in the pathogenesis of IL-13-induced inflammation and remodeling. J Immunol. 2004;172(3):1872-1881.
58. Chiaramonte MG, Donaldson DD, Cheever AW, et al. An IL-13 inhibitor blocks the development of hepatic fibrosis during a T-helper type 2-dominated inflammatory response. J Clin Invest. 1999;104(6):777-785.

59. Zheng T, Zhu Z, Wang Z, et al. Inducible targeting of IL-13 to the adult lung causes matrix metalloproteinase- and cathepsindependent emphysema. J Clin Invest. 2000;106(9):1081-1093.

60. Gonzalo JA, Lloyd CM, Wen D, et al. The coordinated action of $\mathrm{CC}$ chemokines in the lung orchestrates allergic inflammation and airway hyperresponsiveness. J Exp Med. 1998;188(1):157-167.

61. Kunkel SL, Lukacs NW, Strieter RM, et al. The role of chemokines in the immunopathology of pulmonary disease. Forum (Genova). 1999;9(4):339-355.

62. Teran LM. CCL chemokines and asthma. Immunol Today. 2000; 21(5):235-242.

63. Wen T, Besse JA, Mingler MK, et al. Eosinophil adoptive transfer system to directly evaluate pulmonary eosinophil trafficking in vivo. Proc Natl Acad Sci USA. 2013;110(15):6067-6072. 